\title{
Pressure damping, a "billowing" septum, and an eerie silence: perioperative, intermittent obstruction of a mitral valve prosthesis
}

\author{
W Keeble, S M Cobbe
}

Department of

Medical Cardiology,

Royal Infirmary, 10

Alexandra Parade,

Glasgow G31 2ER, UK

W Keeble

S M Cobbe

Correspondence to:

Dr Keeble

Accepted for publication 17 May 2000

\begin{abstract}
This case, involving a 74 year old man who underwent mitral valve and aortic valve replacements, provides detailed insight into the perioperative echocardiographic and haemodynamic changes occurring when a mitral valve prosthesis intermittently obstructs. It illustrates the early sequence of electromechanical dissociation which would lead to cardiac arrest should a tilting disc prosthesis be immobilised in the closed position.

(Heart 2000;84:e6)
\end{abstract}

Keywords: prosthetic valve replacement; echocardiography; intermittent obstruction

- ITU04 12:29PM 28 AUG 98 III MON HR = 75 A = I)
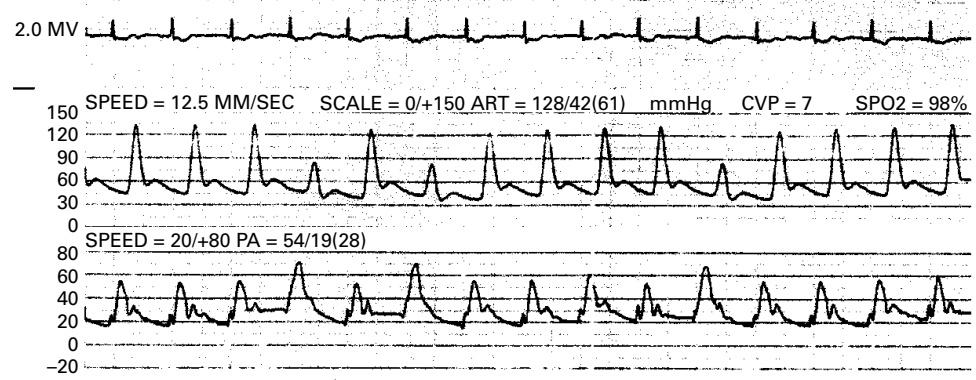

Figure 1 Concurrently recorded ECG, systemic radial arterial pressure, and central pulmonary arterial (PA) pressure tracings. The fourth, sixth, and 11th peak PA pressures step up from $54 \mathrm{~mm} \mathrm{Hg}$ to $70 \mathrm{~mm} \mathrm{Hg}$ but return directly to the base line. Before each peak, the resting diastolic $P A$ pressure is elevated from $19 \mathrm{~mm} \mathrm{Hg}$ to $28 \mathrm{~mm} \mathrm{Hg}$ with a "flat line" appearance. The corresponding fourth, sixth and 11 th radial artery waveforms dampen from $128 / 42 \mathrm{~mm} \mathrm{Hg}$ to $86 / 38 \mathrm{~mm} \mathrm{Hg}$, again returning to the base line. Systemic arterial waveforms appear after the PA waveforms owing to the fractional time delay in transmission between the ascending aorta and measurement at the radial artery. Cardiac rhythm remains regular at 75 beats per minute (paper speed $12.5 \mathrm{~mm} / \mathrm{s}$ ).
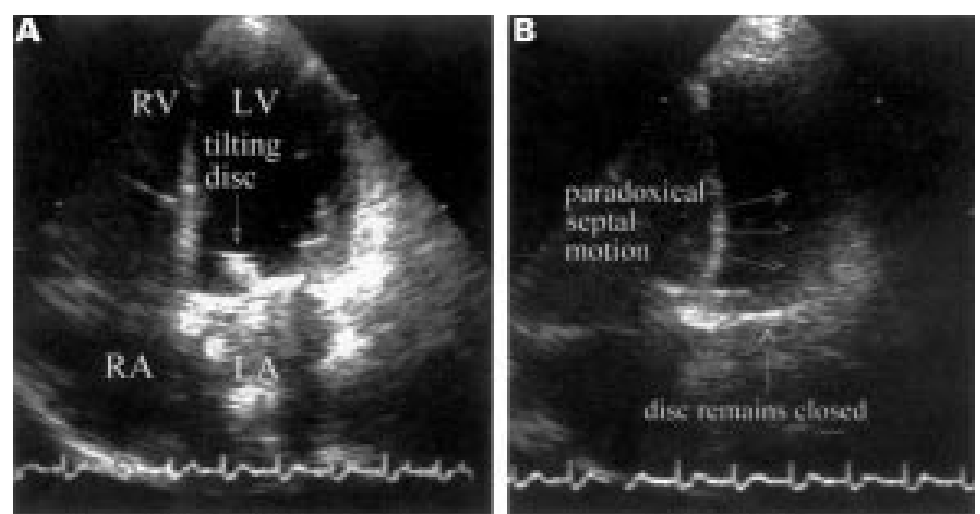

Figure 2 Apical, four chamber, two dimensional views of mitral valve disc prosthesis $(31 \mathrm{~mm})$. (A) The disc pivots appropriately, casting acoustic shadows back into the left atrium. (B) Intermittently, a pronounced "billowing" of the septum occurs into the left ventricle. The prosthetic disc remains in the closed position. LV, left ventricle; $R V$, right ventricle; $L A$, left atrium, $R A$, right atrium.
A 74 year old man underwent uneventful mitral valve ( $31 \mathrm{~mm}$ Ultracor) and aortic valve (27 mm Ultracor) replacements with coronary artery bypass grafting of the left anterior descending artery. Overnight, while ventilated and invasively monitored on the cardiothoracic intensive care unit, intermittent fluctuations in pulmonary arterial (PA) and radial arterial pressures were noted (fig 1).

Two dimensional (cross sectional) echocardiography showed the mitral valve prosthesis functioning appropriately for much of the time, but on occasion the septum would billow violently into the left ventricle (LV) while the prosthesis remained in the closed position (fig 2).

Continuous wave Doppler cutting across both the mitral inflow signal (deflected towards the septum by the prosthesis), and the LV outflow tract signal, confirmed intermittent, abrupt loss of flow into the LV. The flow through the LV outflow tract during ventricular systole would subsequently be reduced on each occasion (fig 3).

By increasing the audio volume on the echo machine and observing the intravascular pressure tracings, the association between silence occurring before each damped radial artery waveform was absolute.

\section{Discussion}

These findings are diagnostic of intermittent mitral valve prosthesis obstruction. An explanation for the interrelation between the echocardiographic and haemodynamic features may be offered. When the disc is jammed closed in ventricular diastole, minimal filling of the LV occurs and the diastolic LV pressure, if measured, would be reduced. In contrast, the diastolic PA and right ventricular pressures rise, since there is no exit of blood from the left atrium. The combination of reduced LV filling and the reversal of ventricular diastolic pressures causes the septum to "billow" momentarily into the LV (fig 2B). Right ventricular systole then causes an excessive elevation of pulmonary arterial pressure. In contrast, the LV contracting on a small ventricular volume generates a diminished pressure wave (fig 1). The gradient of elevated PA pressure and reduced LV pressure is sufficient to free the prosthetic disc. An augmented mitral inflow signal occurs on continuous wave Doppler (fig 3). Normal systemic radial artery and PA pressures are immediately restored. Should the prosthesis jam in the closed position once more, an identical repeat of the situation occurs. 


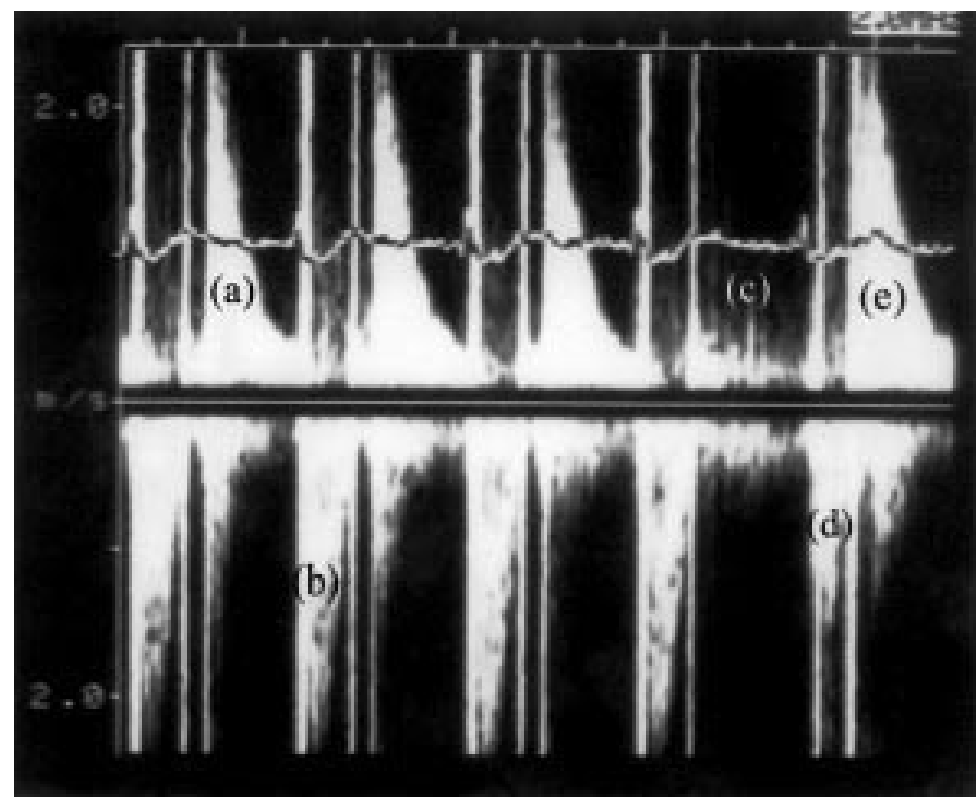

Figure 3 Continuous wave Doppler cutting across both mitral prosthetic inflow signal (a) and the left ventricular outflow tract signal (b). There is abrupt loss of the fourth mitral signal (c), demonstrating absent flow into the left ventricle. Subsequent flow in the left ventricular outflow tract is reduced (d). The fifth mitral inflow signal (e) is augmented.

In the absence of prolonged haemodynamic disturbance, the patient was observed and the fluctuations in pressure tracings gradually resolved within 48 hours. Intermittent obstruction of a mitral prosthesis is extremely rare. The benign outcome in this case suggests a minor piece of debris or remnant of native valve apparatus, not evident on transthoracic echocardiography, was being compressed between the disc edge and valve rim. The intermittent jamming of the disc was evidently self limiting as the tissue was worn down. Late intermittent obstruction of a mitral prosthesis has been recorded, ${ }^{1}$ but in this case, follow up has been unremarkable. Published series of valve replacements reveals disc immobilisation to be a potentially catastrophic but rare event. Three obstructed mitral prostheses complicated a series of 377 valve replacements but were evident immediately on weaning from cardiopulmonary bypass. The cause of obstruction was found to be chordal debris. ${ }^{2}$ In a series of 1645 mitral prosthetic implants, three tilting disc prostheses intermittently obstructed with haemodynamic deterioration within eight hours of surgery and one patient presented with cardiac arrest at 48 hours. $^{3}$ All cases returned to theatre and the obstruction found either to be remnants of chordae, native leaflet, suture or left ventricular wall.

1 Greenwood JP, Nolan J, Mackintosh AF. Late, intermittent obstruction of a mitral prosthesis by chordal remnants. Eur f Cardiothorac Surg 1997;12:804-6.

2 Pai GP, Eleison RG, Rubin JW, et al. Disc immobilization of Bjork-Shiley and Medtronic Hall valves during and immediately after valve replacement. Ann Thorac Surg 1987;44: $73-6$.

3 Sante P, Renzulli A, Festa M, et al. Acute postoperative block of mechanical prostheses: incidence and treatment. Cardiovascular Surgery 1994;2:403-6 